\title{
Perfil audiométrico de trabalhadores do distrito industrial de Maracanaú - CE
}

\section{Audiometric profile of Maracanaú's industrial district workers}

\author{
Renata de Mesquita Teles ${ }^{1}$, Márcia Pinheiro Hortencio de Medeiros ${ }^{2}$
}

\begin{abstract}
RESUMO
Objetivo: Estabelecer o perfil audiométrico de trabalhadores expostos ao ruído, acima do nível de ação (85 dB), em empresas do Distrito Industrial de Maracanaú, Ceará. Métodos: Foi realizado um estudo descritivo de prevalência a partir da avaliação de dados de exames audiométricos, coletados de arquivos das empresas pesquisadas realizados no período de abril a outubro de 2006. Foram avaliados 5372 trabalhadores das $47(52,80 \%)$ empresas que autorizaram a pesquisa e as variáveis analisadas foram: faixa etária, tempo total de exposição ao ruído, estado da acuidade auditiva, tipo de perda auditiva, lateralidade da perda auditiva e configuração audiométrica sugestiva de perda auditiva induzida por ruído nos trabalhadores e setor da atividade econômica e porte da empresa. Resultados: Constatou-se que 19,00\% da população estudada apresentaram alteração, predominando a perda auditiva sensório neural em $90,67 \%$ dos casos. Em $12,71 \%$ da população estudada observou-se quadro sugestivo de perda auditiva induzida por ruído (PAIR) e, em $71,77 \%$ da população portadora de perda auditiva sensório neural, há indícios de PAIR. Em relação à lateralidade da perda, 39,09\% é unilateral, sendo 24,03\% unilateral esquerda. Conclusões: Apesar dos resultados não representarem a prevalência total de perdas auditivas do Distrito, eles apontam para a necessidade não apenas de realização de audiometrias ocupacionais pontuais, mas também de monitoramento da audição dos trabalhadores de forma longitudinal, como parte de um programa de conservação auditiva.
\end{abstract}

DESCRITORES: Perda auditiva; Audiometria; Saúde do trabalhador; Ruído ocupacional; Prevalência

\section{INTRODUÇÃO}

A exposição ocupacional ao ruído é objeto de estudo de pesquisadores de diversas áreas, por ser o risco mais prevalente e o que expõe o maior número de trabalhadores em todo o mundo. $\mathrm{O}$ efeito mais conhecido desta exposição é a perda auditiva induzida por ruído (PAIR) ou perda auditiva induzida por níveis de pressão sonora elevados (PAINPSE), considerada hoje como a doença profissional mais prevalente nos ambientes laborais ${ }^{(1-2-3)}$.

Objetivamente, considera-se como ruído todo sinal acústico aperiódico, originado da superposição de vários movimentos de vibração com diferentes frequiências, as quais não

(1) Fonoaudióloga do Setor de Audiologia do Núcleo de Referência em Saúde Jorge Parente Frota Júnior do Serviço Social da Indústria - SESI - Fortaleza (CE), Brasil; Especialista em Audiologia pela Universidade de Fortaleza UNIFOR - Fortaleza (CE), Brasil.

(2) Mestre, Docente do curso de graduação em Fonoaudiologia da Universidade de Fortaleza - UNIFOR - Fortaleza (CE), Brasil.

Projeto financiado pelo Departamento Nacional para os Programas Estratégicos do Serviço Social da Indústria - SESI - Fortaleza (CE), Brasil, nas áreas de Educação, Lazer e Saúde.

Endereço para correspondência: Renata de Mesquita Teles. R. Bárbara de Alencar, 1442, Aldeota, Fortaleza - CE, CEP 60140-000. E-mail: rtmesquita@sfiec.org.br

Recebido em: 22/3/2007; Aceito em: 30/6/2007 apresentam relação entre $\operatorname{si}^{(4)}$, já subjetivamente, considerase como ruído toda sensação de desagrado, desconforto e/ou de intolerância decorrente de uma exposição sonora ${ }^{(5)}$.

A exposição ao ruído pode provocar diferentes respostas nos trabalhadores de ordem auditiva e extra-auditiva a depender das características do risco, da exposição e do indivíduo exposto. São efeitos auditivos reconhecidos: o zumbido de pitch agudo, a mudança temporária do limiar (MTL) e a mudança permanente do limiar (MPL) (trauma acústico agudo e crônico) e são efeitos extra-auditivos: distúrbios no cérebro e nos sistemas nervoso, circulatório, digestório, endócrino, imunológico, vestibular, muscular, nas funções sexuais e reprodutivas, no psiquismo, no sono, na comunicação e no desempenho de tarefas físicas e mentais ${ }^{(6)}$.

A PAIR é a mudança permanente do limiar decorrente de um trauma acústico crônico e se caracteriza por ser do tipo sensório-neural, geralmente bilateral e simétrica, irreversível, de grau leve nas frequiências baixas e severo nas freqüências altas, com configuração audiométrica típica (entalhe em forma de V) na faixa de freqüências de 6000, 4000 e/ou 3000 $\mathrm{Hz}$, que progride lentamente nas freqüências de 8000, 2000 , 1000,500 e $250 \mathrm{~Hz}$ e atinge seu nível máximo, nas frequiências mais altas, nos primeiros 10 a 15 anos de exposição estável a nível de pressão sonora (NPS) elevados e que interrompe sua progressão uma vez cessada a exposição ${ }^{(7)}$. 
A prevalência de PAIR de origem ocupacional em industriários de um mesmo setor da atividade econômica é extremamente variável tanto em estudos internacionais quanto em nacionais devido a fatores relacionados ao risco, à exposição e aos trabalhadores das populações estudadas. No Brasil, além dos precários sistemas de informação e de fiscalização, temos ainda a questão da sub-notificação, fato que dificulta a determinação da magnitude do problema.

Frente a isto, surgiu o interesse em desenvolver esta pesquisa, que tem como objetivo estabelecer o perfil audiométrico de trabalhadores do maior e mais importante conglomerado industrial do estado do Ceará, localizado no município de Maracanaú, que é o responsável por $10 \%$ do Imposto sobre Circulação de Mercadorias e Serviços (ICMS) arrecadados em todo o estado. Além disso, pretende-se verificar a existência de associação entre as seguintes variáveis: faixa etária, tempo total de exposição ao ruído, estado da acuidade auditiva, tipo de perda auditiva, lateralidade da perda auditiva, configuração audiométrica sugestiva de perda auditiva induzida por ruído nos trabalhadores, setor da atividade econômica e porte da empresa.

\section{MÉTODOS}

O delineamento do estudo foi do tipo individuadoobservacional-seccionado ou de prevalência, realizado a partir da coleta de dados de arquivo das audiometrias de empresas do Distrito Industrial de Maracanaú (CE), no período de abril a outubro de 2006, após a devida aprovação do Comitê de Ética em Pesquisa (CEP) da Universidade de Fortaleza (UNIFOR), sob o no 327/2005 e a assinatura do termo de autorização de fiel depositário por parte do representante legal ou do responsável imediato das empresas.

O Distrito Industrial (DI) de Maracanaú foi fundado há 40 anos pelo então governador Sr. Virgílio Távora e em 2005 concentrava 108 empresas de vários setores da atividade econômica dos mais variados portes ${ }^{(8)}$.

Para a composição da amostra foram estabelecidos os seguintes critérios de inclusão: empresas localizadas em Maracanaú (CE) que assinaram o termo de autorização de fiel depositário, que expõem seus trabalhadores a níveis de pressão sonora ou doses superiores aos níveis de ação recomendados na NR-9 - Programa de Prevenção de Riscos Ambientais (PPRA), respectivamente 80 dBNEN(A) e 50\% e que realizam audiometria nos expostos e trabalhadores que se encontram na ativa com ou sem história atual ou pregressa de exposição ao ruído ocupacional.

A amostra foi constituída quanto ao número e percentual de empresas que preencheram os critérios de inclusão: 89 $(82,40 \%)$. Porém, apenas $47(52,80 \%)$ participaram do estudo, pois as empresas assinaram o termo de autorização de fiel depositário; quanto ao percentual de empresas pesquisadas por setor de atividade econômica: metalúrgico $07(53,85 \%)$, tecelagem 07 (46,15\%), calçadista $01(100 \%)$, químico $02(18,18 \%)$, plástico $02(40 \%)$, siderúrgico 02 (100\%), couro $01(100 \%)$, produtos minerais $05(83,33 \%)$, alimentício $01(16,67 \%)$, bebidas $03(100 \%)$, celulose 03 (100\%), eletroeletrônico 02 (66,67\%), madeireiro 01 (50\%), confecção $05(57,14 \%)$, serviços $02(66,67 \%)$ e outras 03 (100\%); quanto ao número e percentual de empresas pesquisadas por porte: micro $07(35 \%)$, pequeno $25(70,27 \%)$, médio 14 (54,55\%) e grande $01(25 \%)$; quanto ao número e percentual de trabalhadores expostos pesquisados em relação ao total de trabalhadores expostos e não-expostos das empresas: 5372 (45,15\%); quanto ao percentual de trabalhadores expostos pesquisados por porte da empresa em relação ao total de trabalhadores expostos e não-expostos: micro: $(56,15 \%)$, pequeno: $(92,45 \%)$, médio: $(58,20 \%)$ e grande: $(13,15 \%)$; quanto ao gênero: masculino $4.413(84,10 \%)$ e feminino 837 (15,9\%); quanto à idade: mínima (15 anos), mediana (32 anos) e máxima (86 anos) e quanto ao tempo de exposição total ao ruído: mínimo ( 0 ano), mediano (5,2 anos) e máximo (44 anos).

A coleta de dados foi realizada a partir das medições de ruído do PPRA e do único (nos casos pré-admissionais) ou do último exame audiométrico (nos casos de periódicos) dos trabalhadores das empresas que preenchiam os critérios de inclusão e que assinaram o termo de autorização. Foram variáveis analisadas neste estudo: faixa etária (15-25, 26-35, $36-45, \geq 46$ anos), faixa de tempo de exposição total ao ruído (0-10, 11-20 e $\geq 21$ anos), setor da atividade econômica (alimentício, madeireiro, metalúrgico, químico, têxtil, plástico, confecção, serviços, bebidas, eletro eletrônico, calçado, celulose, couro, produtos minerais, siderúrgico e indústrias diversas), porte da empresa (micro 0-19; pequeno 20-99; médio 100-499 e grande porte a partir de 500 funcionários), estado da acuidade auditiva (normal: limiares aéreos de -10 a 25 dBNA e alterado: limiares aéreos $>25$ dBNA), tipo de perda auditiva (condutiva/PAC: apenas VA alterada e gap A$\mathrm{O} \geq 15 \mathrm{dBNA}$, sensório-neural/PASN VA e VO alteradas e gap A-O $\leq 10$ dBNA e mista/PAM: VA e VO alteradas e gap $\mathrm{A}-\mathrm{O} \geq 15$ dBNA), lateralidade da perda auditiva (bilateral e unilateral (direita e esquerda)) e configuração audiométrica sugestiva de PAIR (sim e não) conforme caracterização do Comitê Nacional de Ruído e Conservação Auditiva CONARCA (1999).

Os dados foram digitados em um banco de dados, analisados através da estatística descritiva no programa SPSS 8.0 for Windows e apresentados em tabelas.

\section{RESULTADOS}

Na Tabela 1 dos 5372 trabalhadores pesquisados, constatou-se um percentual de 19\% de perda auditiva. Dos 1019 trabalhadores com perda auditiva, predominou a ocorrência de PASN com 924 (90,67\%) casos em detrimento a ocorrência de PAC e PAM somadas. O setor têxtil foi o que apresentou o maior número de registros (1531) e o alimentício o menor (15). O setor alimentício foi o que apresentou os maiores percentuais tanto de PASN quanto de PAC somada a PAM, o que não pode ser levado em consideração devido à pequena quantidade de registros nesse setor. Através do teste do Qui-quadrado de Pearson, verificou-se que os percentuais de PASN não diferem significativamente $(\mathrm{p}>0,05)$ entre os setores de bebida, calçado, couro, eletroeletrônico, metalúrgico, produtos minerais, serviços e celulose, nem entre 
os setores têxtil, siderúrgico, indústrias diversas e confecção e nem entre os setores plástico e químico, mas diferem significativamente $(\mathrm{p}<0,05)$ entre esses três grupos. Para o teste foram excluídos os setores alimentício e químico em função do pequeno número de registros. A variável setor da atividade econômica, isoladamente, não apresentou significância estatística $(\mathrm{p}<0,05)$.

Tabela 1. Distribuição da situação auditiva dos trabalhadores em função do setor da atividade econômica da empresa

\begin{tabular}{|c|c|c|c|c|c|}
\hline Setor & & Normal & PASN & PAC+PAM & Total \\
\hline \multirow[t]{2}{*}{ Confecção } & $\mathrm{N}$ & 645 & 85 & 15 & 745 \\
\hline & $\%$ & $86,60 \%$ & $11,40 \%$ & $2,00 \%$ & $100,00 \%$ \\
\hline \multirow[t]{2}{*}{ Siderúrgico } & $\mathrm{N}$ & 255 & 36 & 2 & 293 \\
\hline & $\%$ & $87,00 \%$ & $12,30 \%$ & $0,70 \%$ & $100,00 \%$ \\
\hline \multirow[t]{2}{*}{ Têxtil } & $\mathrm{N}$ & 1304 & 201 & 26 & 1531 \\
\hline & $\%$ & $85,20 \%$ & $13,10 \%$ & $1,70 \%$ & $100,00 \%$ \\
\hline \multirow[t]{2}{*}{ Indústrias Diversas } & $\mathrm{N}$ & 51 & 8 & $\longrightarrow$ & 59 \\
\hline & $\%$ & $86,40 \%$ & $13,60 \%$ & $\longrightarrow$ & $100,00 \%$ \\
\hline \multirow[t]{2}{*}{ Serviços } & $\mathrm{N}$ & 39 & 8 & 1 & 48 \\
\hline & $\%$ & $81,30 \%$ & $16,70 \%$ & $2,10 \%$ & $100,00 \%$ \\
\hline \multirow[t]{2}{*}{ Couro } & $\mathrm{N}$ & 287 & 64 & 3 & 354 \\
\hline & $\%$ & $81,10 \%$ & $18,10 \%$ & $0,80 \%$ & $100,00 \%$ \\
\hline \multirow[t]{2}{*}{ Celulose } & $\mathrm{N}$ & 209 & 51 & 10 & 270 \\
\hline & $\%$ & $77,40 \%$ & $18,90 \%$ & $3,70 \%$ & $100,00 \%$ \\
\hline \multirow[t]{2}{*}{ Metalúrgico } & $\mathrm{N}$ & 450 & 109 & 13 & 572 \\
\hline & $\%$ & $78,70 \%$ & $19,10 \%$ & $2,30 \%$ & $100,00 \%$ \\
\hline \multirow[t]{2}{*}{ Bebidas } & $\mathrm{N}$ & 429 & 115 & 7 & 551 \\
\hline & $\%$ & $77,90 \%$ & $20,90 \%$ & $1,30 \%$ & $100,00 \%$ \\
\hline \multirow[t]{2}{*}{ Calçado } & $\mathrm{N}$ & 50 & 14 & 2 & 66 \\
\hline & $\%$ & $75,80 \%$ & $21,20 \%$ & $3,00 \%$ & $100,00 \%$ \\
\hline \multirow[t]{2}{*}{ Eletro eletrônico } & $\mathrm{N}$ & 115 & 34 & 3 & 152 \\
\hline & $\%$ & $75,70 \%$ & $22,40 \%$ & $2,00 \%$ & $100,00 \%$ \\
\hline \multirow[t]{2}{*}{ Madereiro } & $\mathrm{N}$ & 18 & 6 & 1 & 25 \\
\hline & $\%$ & $72,00 \%$ & $24,00 \%$ & $4,00 \%$ & $100,00 \%$ \\
\hline \multirow[t]{2}{*}{ Produtos Minerais } & $\mathrm{N}$ & 322 & 106 & 6 & 434 \\
\hline & $\%$ & $74,20 \%$ & $24,40 \%$ & $1,40 \%$ & $100,00 \%$ \\
\hline \multirow[t]{2}{*}{ Plástico } & $\mathrm{N}$ & 66 & 30 & 2 & 98 \\
\hline & $\%$ & $67,30 \%$ & $30,60 \%$ & $2,00 \%$ & $100,00 \%$ \\
\hline \multirow[t]{2}{*}{ Químico } & $\mathrm{N}$ & 105 & 52 & 2 & 159 \\
\hline & $\%$ & $66,00 \%$ & $32,70 \%$ & $1,30 \%$ & $100,00 \%$ \\
\hline \multirow[t]{2}{*}{ Alimentício } & $\mathrm{N}$ & 8 & 5 & 2 & 15 \\
\hline & $\%$ & $53,30 \%$ & $33,30 \%$ & $13,30 \%$ & $100,00 \%$ \\
\hline \multirow[t]{2}{*}{ Total } & $\mathrm{N}$ & 4353 & 924 & 95 & 5372 \\
\hline & $\%$ & $81,00 \%$ & $17,20 \%$ & $1,80 \%$ & $100,00 \%$ \\
\hline
\end{tabular}

Teste Qui-quadrado de Pearson

Legenda: PASN - Perda auditiva sensório neural; PAC - Perda auditiva condutiva; PAM - perda auditiva mista

Na Tabela 2 constatou-se o maior percentual de audiometrias alteradas nas empresas de micro porte $(31,50 \%)$, tanto para a PASN quanto para a PAC somada a PAM. O médio porte foi o que apresentou o maior número de registros (2945) e o micro porte o menor (105). Através do teste do Qui-quadrado de Pearson, verificou-se que os percentuais de PASN não diferem significativamente $(\mathrm{p}<0,05)$ entre as empresas de porte grande e médio, nem entre as empresas de pequeno e micro porte, mas diferem significativamente $(\mathrm{p}<0,05)$ entre dois grupos (grande-médio e pequeno-micro). A variável porte da empresa, isoladamente, não apresentou significância estatística $(\mathrm{p}<0,05)$.

Tabela 2. Distribuição da situação auditiva dos trabalhadores em função do porte da empresa

\begin{tabular}{lccccc}
\hline Porte & & Normal & PASN & PAC+PAM & Total \\
\hline Grande & $\mathrm{N}$ & 519 & 86 & 16 & 621 \\
& $\%$ & $83,60 \%$ & $13,80 \%$ & $2,60 \%$ & $100,00 \%$ \\
Médio & $\mathrm{N}$ & 2459 & 448 & 38 & 2945 \\
& $\%$ & $83,50 \%$ & $15,20 \%$ & $1,30 \%$ & $100,00 \%$ \\
Pequeno & $\mathrm{N}$ & 1303 & 362 & 36 & 1701 \\
& $\%$ & $76,60 \%$ & $21,30 \%$ & $2,10 \%$ & $100,00 \%$ \\
Micro & $\mathrm{N}$ & 72 & 28 & 5 & 105 \\
& $\%$ & $68,60 \%$ & $26,70 \%$ & $4,80 \%$ & $100,00 \%$ \\
\hline Total & $\mathrm{N}$ & 4353 & 924 & 95 & 5372 \\
& $\%$ & $81,00 \%$ & $17,20 \%$ & $1,80 \%$ & $100,00 \%$ \\
\hline
\end{tabular}

Teste Qui-quadrado de Pearson

Legenda: PASN - Perda auditiva sensório neural; PAC - Perda auditiva condutiva; PAM - perda auditiva mista

$\mathrm{Na}$ Tabela 3 constatou-se que os maiores percentuais de PASN obtidos foram no gênero masculino e na orelha esquerda. $\mathrm{O}$ gênero masculino teve o maior número de registro (4413), o que corresponde a $84,10 \%$ do total de trabalhadores pesquisado. De acordo com teste Qui-quadrado de Pearson a variável gênero, isoladamente, não apresentou significância estatística $(\mathrm{p}<0,05)$.

Tabela 3. Distribuição da situação auditiva dos trabalhadores em função do gênero

\begin{tabular}{llcccc}
\hline Gênero & & Normal & PASN & PAC+PAM & Total \\
\hline Feminino & OD & 795 & 30 & 12 & 837 \\
& $\%$ & $15,10 \%$ & $0,60 \%$ & $0,20 \%$ & \\
& OE & 788 & 40 & 09 & $15,90 \%$ \\
Masculino & $\%$ & $15,00 \%$ & $0,80 \%$ & $0,20 \%$ & \\
& OD & 3781 & 578 & 54 & 4413 \\
& $\%$ & $72,00 \%$ & $11,00 \%$ & $1,00 \%$ & \\
& OE & 3687 & 664 & 62 & $84,10 \%$ \\
& $\%$ & $70,2 \%$ & $12,60 \%$ & $1,00 \%$ & \\
\hline
\end{tabular}

Teste Qui-quadrado de Pearson

Legenda: PASN - Perda auditiva sensório neural; PAC - Perda auditiva condutiva; PAM - perda auditiva mista; OD- orelha direita; OE - orelha esquerda.

Na Tabela 4 os maiores percentuais de audiometrias alteradas e de PASN foram constatados na faixa etária acima de 45 anos com 302 casos $(48,80 \%)$ e 292 casos $(47,20 \%)$, respectivamente. O maior número de registros ocorreu na faixa etária de 25-35 anos (2094) e o menor na faixa etária acima de 45 anos (618). Através do teste do Qui-quadrado de Pearson, verificou-se que os percentuais de PASN diferem significativamente $(\mathrm{p}<0,05)$ entre as faixas etárias de 15 a 25 anos (referência), de 25 a 35 anos, de 35 a 45 anos e acima de 45 anos. A variável faixa etária apresentou significância estatística $(\mathrm{p}<0,05)$. 
Tabela 4. Distribuição da situação auditiva dos trabalhadores em função da faixa etária

\begin{tabular}{lccccc}
\hline Faixa etária & & Normal & PASN & PAC+PAM & Total \\
\hline $15|-| 25$ anos & $\mathrm{N}$ & 1142 & 78 & 17 & 1237 \\
& $\%$ & $92,30 \%$ & $6,30 \%$ & $1,40 \%$ & $100,00 \%$ \\
$25-\mid 35$ anos & $\mathrm{N}$ & 1827 & 230 & 37 & 2094 \\
& $\%$ & $87,20 \%$ & $11,00 \%$ & $1,80 \%$ & $100,00 \%$ \\
$35-\mid 45$ anos & $\mathrm{N}$ & 1015 & 315 & 29 & 1359 \\
& $\%$ & $74,70 \%$ & $23,20 \%$ & $2,10 \%$ & $100,00 \%$ \\
acima de 45 anos & $\mathrm{N}$ & 316 & 292 & 10 & 618 \\
& $\%$ & $51,10 \%$ & $47,20 \%$ & $1,60 \%$ & $100,00 \%$ \\
\hline Total & $\mathrm{N}$ & 4300 & 915 & 93 & 5308 \\
& $\%$ & $81,00 \%$ & $17,20 \%$ & $1,80 \%$ & $100,00 \%$ \\
\hline
\end{tabular}

Teste Qui-quadrado de Pearson

Legenda: PASN - Perda auditiva sensório neural; PAC - Perda auditiva condutiva; PAM - perda auditiva mista

Na Tabela 5 os maiores percentuais de audiometrias alteradas e PASN foram encontrados nos trabalhadores expostos ao ruído acima de 20 anos com 90 casos $(55,50 \%)$ e com 83 casos $(51,20 \%)$, respectivamente. O maior número de registros ocorreu na faixa de tempo de exposição 0-10 anos (3130) e o menor na faixa de tempo acima de 20 anos (162). Através do teste do Qui-quadrado de Pearson, verificou-se que os percentuais de PASN diferem significativamente $(\mathrm{p}<0,05)$ entre as faixas de 0 a 10 anos, acima de 10 a 20 anos e acima de 20 anos. A variável tempo total de exposição ao ruído ocupacional apresentou significância estatística $(\mathrm{p}<0,05)$.

Tabela 5. Distribuição da situação auditiva dos trabalhadores em função do tempo total de exposição ao ruído ocupacional

\begin{tabular}{lccccc}
\hline $\begin{array}{l}\text { Tempo total } \\
\text { de exposição }\end{array}$ & & Normal & PASN & PAC+PAM & Total \\
\hline $0-10$ anos & $\mathrm{N}$ & 2630 & 448 & 52 & 3130 \\
& $\%$ & $84,00 \%$ & $14,30 \%$ & $1,70 \%$ & $100,00 \%$ \\
$10-\mid 20$ anos & $\mathrm{N}$ & 514 & 235 & 16 & 765 \\
& $\%$ & $67,20 \%$ & $30,70 \%$ & $2,10 \%$ & $100,00 \%$ \\
acima de 20 anos & $\mathrm{N}$ & 72 & 83 & 7 & 162 \\
& $\%$ & $44,40 \%$ & $51,20 \%$ & $4,30 \%$ & $100,00 \%$ \\
\hline Total & $\mathrm{N}$ & 3216 & 766 & 75 & 4057 \\
& $\%$ & $79,30 \%$ & $18,90 \%$ & $1,80 \%$ & $100,00 \%$ \\
\hline
\end{tabular}

Teste Qui-quadrado de Pearson

Legenda: PASN - Perda auditiva sensório neural; PAC - Perda auditiva condutiva; PAM - perda auditiva mista

Na Tabela 6 constatou-se uma prevalência de $12,71 \%$ de PAIR na população total de trabalhadores e de $71,67 \%$ na população de trabalhadores com PASN.

$\mathrm{Na}$ Tabela 7 constatou-se que os maiores percentuais de trabalhadores com PASN sugestivas de PAIR tiveram acometimento unilateral $(38,09 \%)$, seguido do bilateral $(33,58 \%)$ e dos trabalhadores com PASN sugestivas de PAIR unilateral, predominou o acometimento unilateral esquerdo $(24,03 \%)$.
Tabela 6. Distribuição dos trabalhadores com PASN sugestiva de PAIR

\begin{tabular}{lccc}
\hline PAIR & N & $\%$ total & $\%$ PASN \\
\hline PASN não sugestiva de PAIR & 270 & $5,03 \%$ & $28,33 \%$ \\
PASN sugestiva de PAIR & 683 & $12,71 \%$ & $71,67 \%$ \\
Subtotal & 953 & $17,74 \%$ & $100,00 \%$ \\
Normal + Outras Perdas & 4419 & $82,26 \%$ & \\
\hline Total & 5372 & $100,00 \%$ & \\
\hline
\end{tabular}

Legenda: PASN - Perda auditiva sensório neural; PAIR - perda auditiva induzida por ruído.

Tabela 7. Distribuição dos trabalhadores com PASN sugestiva de PAIR segundo a lateralidade

\begin{tabular}{lccc}
\hline PAIR & N & $\%$ total & $\%$ PAIR \\
\hline Não sugestiva de PAIR & 270 & $5,03 \%$ & $28,33 \%$ \\
Sugestiva de PAIR OD & 134 & $2,49 \%$ & $14,06 \%$ \\
Sugestiva de PAIR OE & 229 & $4,26 \%$ & $24,03 \%$ \\
Sugestiva de PAIR AO & 320 & $5,96 \%$ & $33,58 \%$ \\
Subtotal & 953 & $17,74 \%$ & $100,00 \%$ \\
Normal + PAC + PAM & 4419 & $82,26 \%$ & \\
\hline Total & 5372 & $100,00 \%$ & \\
\hline
\end{tabular}

Legenda: PASN - Perda auditiva sensório neural; PAC - perda auditiva condutiva; PAM - perda auditiva mista; PAIR - perda auditiva induzida por ruído; AO - ambas orelhas.; OD - orelha direita; OE - orelha esquerda.

\section{DISCUSSÃO}

Uma limitação deste estudo foi a não autorização da coleta de dados por parte do representante legal ou do representante imediato de $42(47,2 \%)$ das 89 empresas que preenchiam os critérios de inclusão do Distrito Industrial de Maracanaú (CE). As empresas não participantes quanto ao número de empresas por setor foram: 06 metalúrgico, 07 têxtil, 06 alimentício, 01 eletro eletrônico, 01 madeireiro, 03 plástico, 01 produtos minerais, 09 químico, 05 confecção e 03 serviços e quanto ao número de empresas por porte não autorizadas, foram: grande porte (03), médio porte (14), pequeno porte (16) e micro porte (15).

Não conseguimos localizar muitos estudos nacionais sobre a prevalência de PASN em trabalhadores de diversos setores industriais, a maioria concentra-se na prevalência de PAIR em uma ou em algumas empresas de um setor específico. Em uma dissertação de mestrado ${ }^{(9)}$ foi pesquisado o perfil audiométrico de trabalhadores de empresas de vários setores da atividade econômica da cidade de Fortaleza e região metropolitana, constatando $26,4 \%$ de perdas auditivas, sendo 93,1\% do tipo sensório-neural. Em um estudo semelhante realizado em trabalhadores de empresas da cidade de Salvador e região metropolitana, constatou a alarmante prevalência de $45,9 \%$ de perda auditiva, com maior ocorrência nos setores gráfico $(58,7 \%)$, mecânico $(51,7 \%)$, bebida $(45,9 \%)$, químico/petroquímico $(42,3 \%)$ e metalúrgico $(35,8 \%)^{(10)}$. Foram analisadas as audiometrias de 741 trabalhadores de quatro metalúrgicas de Piracicaba (SP), constando $41 \%$ de perdas auditivas ${ }^{(11)}$. Analisaram exames audiométricos pré- 
admissionais de 3.117 candidatos a vaga em vários setores industriais de Campinas (SP) e constataram 19,6\% de perda auditiva, sendo $65,7 \%$ de PASN na orelha direita e $79,7 \%$ de PASN na orelha esquerda ${ }^{(12)}$. As diferenças nas prevalências de perda auditiva entre os estudos supracitados e esse estudo (Tabela 1), decorrem das diferentes características das populações estudadas quanto a idade, tempo de exposição, etc. Em seus respectivos estudos constataram que a maior parte das PASN em trabalhadores ou são sugestivas de PAIR ou são PAIR ocupacionais já diagnosticadas ${ }^{(9-10)}$.

A ocorrência dos maiores percentuais de prevalência de perdas auditivas em trabalhadores de empresas de micro-porte (Tabela 2), talvez se deva ao fato dessas empresas não serem os maiores alvos de fiscalização da DRT e por conta disso, não investem na implementação de programas nas áreas de saúde e segurança do trabalho, apesar da exigência legal, e quando realizam exames audiométricos ocupacionais, os fazem desconectados do programa de conservação auditiva. Outro fato a considerar, seria a escassez de recursos financeiros para à micro e pequena empresa. Não foram encontrados na literatura estudos relativos ao porte da empresa.

A maioria dos estudos concentra-se na prevalência de PAIR entre os gêneros, ressaltando uma maior ocorrência de PAIR no gênero masculino, provavelmente devido a maior exposição dos homens a ruídos ocupacionais e extraocupacionais $^{(13-14)}$. Como a maioria das PASN ou são sugestivas de PAIR ou são PAIR ocupacionais diagnosticadas, pode-se utilizar a mesma explicação para o fato da maior ocorrência de PASN no gênero masculino (Tabela 3).

Os maiores percentuais (Tabela 4) de audiometrias alteradas e de PASN foram constatados na faixa etária acima de 45 anos, faixa etária onde tem início o desgaste natural da audição chamado de presbiacusia ${ }^{(15)}$, que se caracteriza por uma PASN, progressiva, em freqüências altas, com predominância em $8 \mathrm{KHz}$ e mais acentuada no gênero masculino ${ }^{(9)}$. Como a maioria das PASN ou são sugestivas de PAIR ou são PAIR ocupacionais já diagnosticadas, pode-se utilizar a mesma explicação para o fato da maior ocorrência de PASN com o aumento da idade. Nas pesquisas ${ }^{(9,16-17)}$ o fator idade também se associou à PAIR, constatando um aumento da prevalência de PAIR com o aumento da idade. Em seu estudo constatou uma associação estatisticamente significativa entre o fator idade e a PAIR e um aumento do risco para a PAIR com o avanço da idade com maior risco na faixa etária de 48-72 anos ${ }^{(9)}$. Em uma pesquisa realizada com 222 trabalhadores do sexo masculino acometidos por PASN sugestiva de PAIR ocupacional encaminhados ao Serviço Médico de Saúde do Trabalhador pertencente ao Serviço Social da Indústria (SESI), em Vila Leopoldina, em São Paulo, constataram que a proporção de queixa clínica de perda auditiva aumentava com a idade e que as alterações audiométricas típicas variavam de acordo com a faixa etária ${ }^{(18)}$.

A Tabela 5 constatou uma maior prevalência de audiometrias alteradas e de PASN em trabalhadores expostos ao ruído acima de 20 anos de tempo total de exposição ao ruído. Em seus estudos, constataram uma maior ocorrência de PASN e PAIR, respectivamente, com o aumento do tempo total de exposição ao ruído ${ }^{(9,16,19)}$. O CONARCA informa que os primeiros 10 a 15 anos de exposição ocupacional ao ruído são críticos para a instalação da PAIR ${ }^{(7)}$. Relata-se um maior número de casos de PAIR em trabalhadores com tempo total de exposição ocupacional ao ruído em torno de 14 anos $^{(20)}$. Em uma pesquisa realizada com 222 trabalhadores do sexo masculino acometidos por PASN sugestiva de PAIR ocupacional encaminhados ao Serviço Médico de Saúde do Trabalhador pertencente ao Serviço Social da Indústria (SESI), em Vila Leopoldina, São Paulo, constataram que a proporção de queixa clínica de perda auditiva aumentava com a evolução do tempo de exposição e que as alterações audiométricas típicas variavam de acordo com o tempo de exposiçãa ${ }^{(18)}$.

Na Tabela 6 constatou-se uma menor prevalência de PAIR na população total de trabalhadores de setores variados quando comparada a outros estudos ${ }^{(9-10,16)}$ o que pode ser explicado devido às diferentes características das populações estudadas quanto à idade e tempo total de exposição ao ruído ocupacional. A maioria das PASN apresentou configuração audiométrica sugestiva de PAIR, o que foi encontrado por vários estudos $^{(9-10)}$. A exposição ao ruído ocupacional é considerada hoje a principal causa de PASN em adultos.

$\mathrm{Na}$ Tabela 7 constatou-se que os maiores percentuais de trabalhadores com PASN sugestivas de PAIR tiveram acometimento unilateral, seguido do bilateral confirmando os achados de outros pesquisadores ${ }^{(9-10)}$ e indo contra a afirmação do CONARCA de que a PAIR é geralmente bilateral e simétrica. A assimetria auditiva pode comprometer a função de localização sonora, o que pode ser vital em alguns setores da atividade econômica. $\mathrm{O}$ posicionamento da fonte sonora em relação à orelha do trabalhador deve ser investigado em futuros estudos. Dos trabalhadores com PASN sugestivas de PAIR unilateral, predominou o acometimento unilateral esquerdo, confirmando os achados de outros pesquisadores ${ }^{(9-10)}$. Os limiares audiométricos das orelhas esquerdas demonstraram serem mais elevados os da direita em estudos realizados tanto em populações de expostos $^{(15)}$ quanto de não-expostos ao ruído.

\section{CONCLUSÃO}

Constatou-se que dos 5372 trabalhadores avaliados, 1019 apresentaram algum tipo de perda auditiva, o que corresponde a 19\%. Dessas alterações 90,67\% são do tipo sensório neural, ou seja, compatíveis à exposição ao ruído, em detrimento a ocorrência de perda auditiva condutiva e mista somadas.

Outro aspecto relevante foi a predominância de alteração auditiva na micro $(31,50 \%)$ e mesmo na pequena indústria $(23,5 \%)$, com resultados estatisticamente semelhantes. Questiona-se aqui se há uma real preocupação com a saúde desses trabalhadores. Sugerem-se programas voltados para esses portes industriais, tão carentes de assistência preventiva bem como, ações que possibilitem a conscientização dos malefícios do ruído e a prática de medidas de prevenção.

Os maiores percentuais de PASN obtidos foram nos trabalhadores do gênero masculino e na orelha esquerda; a faixa etária mais atingida foi acima de 45 anos com 302 casos (48,80\%) para alguma alteração auditiva e 292 casos $(47,20 \%)$, para a perda auditiva sensório neural. 
Os maiores percentuais de perdas auditivas e PASN foram encontrados nos trabalhadores expostos ao ruído acima de 20 anos com 90 casos $(55,50 \%)$ e com 83 casos $(51,20 \%)$, respectivamente.

Uma prevalência de $12,71 \%$ de PAIR na população total de trabalhadores e de 71,67\% na população de trabalhadores com PASN. Os maiores percentuais de trabalhadores com PASN sugestivas de PAIR tiveram acometimento unilateral (38,09\%), seguido do bilateral (33,58\%). Dos trabalhadores com PASN sugestivas de PAIR unilateral, predominou o acometimento unilateral esquerdo $(24,03 \%)$.

Apesar dos resultados obtidos nesse estudo não representarem a prevalência total de perdas auditivas do Distrito Industrial de Maracanaú (CE), eles apontam para a necessidade não apenas de realizar audiometrias ocupacionais pontuais, mas de monitorar a audição dos trabalhadores longitudinalmente por meio de um programa de conservação auditiva.

\section{AGRADECIMENTOS}

Ao SESI - CE, em especial ao Núcleo de Referência em Saúde e ao Departamento Nacional pelo incentivo e apoio financeiro; às empresas que autorizaram a pesquisa; ao LEMA na pessoa do Professor Robson, pela consultoria estatística; à AEDI pelo apoio e reconhecimento; à equipe do CEDIP pela elaboração do material de divulgação; ao Plínio Araújo pelo apoio na elaboração do projeto.

\begin{abstract}
Purpose: To establish the audiometric profile of workers exposed to noise above the action level $(85 \mathrm{~dB})$ in Maracanaú's Industrial District, Ceará (Brazil). Methods: A descriptive study of prevalence was carried out through the evaluation of audiometric data gathered from the files of the researched companies between April and October of 2006 . There were 5372 workers evaluated, from the $47(52,80 \%)$ companies that authorized the study. The studied variables were: age range, total period of exposition to noise, auditory acuity status, type of hearing loss, hearing loss laterality and audiometric configuration suggestive of noise-induced hearing loss (NIHL) in workers, economic activity trade and size of the company. Results: It was observed that $19 \%$ of the studied population presented alterations, with predominance of sensory neural hearing loss (SNHL) in $90.67 \%$ of the cases. In $12.71 \%$ of the population suggestive signs of noise-induced hearing loss (NIHL) were observed, and in $71.77 \%$ of the population with SNHL there were signs of NIHL. Regarding laterality of the hearing loss, $39.09 \%$ was unilateral, from which $24.03 \%$ was on the left ear. Conclusions: Although the results do not represent the total prevalence of hearing loss in the District, they point out to the necessity not only to perform occupational audiometry, but also to longitudinally monitor the hearing of these workers as part of a hearing conservation program.
\end{abstract}

KEYWORDS: Hearing loss; Audiometry; Occupational health; Occupational noise; Prevalence.

\title{
REFERÊNCIAS
}

1. Kwitko A. Os pontos frágeis: A PAIRO está recebendo atenção especial, pois é a mais prevalente doença profissional. Rev Proteção. 1998;73:305 .

2. Lacerda ABM, Stival J. Análise da eficácia de um programa de prevenção da perda auditiva em uma indústria madeireira do ponto de vista fonoaudiológico. J Bras Fonoaudiol. 2001;1:192-7.

3. Guida HL. Efeitos psicossociais da perda auditiva induzida pelo ruído em ex-funcionários da indústria. Acta ORL. 2007;25(1):78-33.

4. Feldman AS, Grimes CT, editors. Hearing conservation in industry. Baltimore: Williams \& Wilkins; 1985.

5. Araújo SA. Perda auditiva induzida pelo ruído em trabalhadores de metalúrgica. Rev Bras Otorrinolaringol. 2002;68(1):47-52.

6. Fernandes M. Estudos dos efeitos auditivos e extra-auditivos da exposição ocupacional a ruído e vibração. Rev Bras Otorrinolaringol. 2002;68(5):705-13.

7. Comitê Nacional de Ruído e Conservação Auditiva (CONARCA). Perda auditiva induzida pelo ruído relacionado ao trabalho. Bol. $\mathrm{n}^{\circ} 1$. São Paulo, 29/06/94.

8. Albuquerque N. Distrito Industrial: 40 anos: Maracanaú - Ceará. Fortaleza: Nazacom; 2004.

9. Medeiros MPH. Perfil audiométrico, prevalência e fatores preditores da perda auditiva induzida por ruído ocupacional [tese]. Fortaleza: Universidade Federal do Ceará; 2001.

10. Miranda CR, Dias CR, Pena PGL, Nobre LCC, Aquino R. Perda auditiva induzida pelo ruído em trabalhadores industriais da Região Metropolitana de Salvador, Bahia. Inf Epidemiol SUS. 1998;7(1):87-94.

11. Gonçalves CGO, Iguti AM. Análise de programas de preservação da audição em quatro indústrias metalúrgicas de Piracicaba, São Paulo, Brasil. Cad Saúde Pública. 2006;22(3):609-18.

12. Franco ES, Russo ICP. Prevalência de perdas auditivas em trabalhadores no processo admissional em empresas na região de Campinas/SP. Rev Bras Otorrinolaringol. 2001;67(5):511-23.

13. Henderson D, Subramaniam M, Boettcher FA. Individual susceptibility to noise-induced hearing loss: an old topic revisited. Ear Hear. 1993;14(3):152-68.

14. Kryter KD. Presbycusis, sociocusis and nosocusis. J Acoust Soc Am. 1983;73:1897-917.

15. International Organization for Standardization. Acoustics (ISO). Determination of occupational noise exposure and estimation of noiseinduced hearing impairment, ISO 1999. Geneve; 1990.

16. Guerra MR, Lourenço BMC, Bustamante-Teixeira MT, Alves MJM. Prevalência de perda auditiva induzida por ruído em empresa metalúrgica. Rev Saúde Pública = J Public Health. 2005;39(2):238-44.

17. Kasper KCF, Gómez MVSG, Zaher VL. O ruído como fator estressante na vida de trabalhadores dos setores de serralheria e marcenaria. Arq Int Otorrinolaringol. 2005;9(1):302-12.

18. Almeida SIC, Albernaz PLM, Zaia PA, Xavier OG, Karazawa EHI. História natural da perda auditiva ocupacional provocada por ruído. Rev 
Assoc Med Bras (1992). 2000;46(2):143-58.

19. Caldart AU, Adriano CF, Terruel I, Martins RF, Caldart AU, Mocellin M. Prevalência da perda auditiva induzida pelo ruído em trabalhadores da indústria têxtil. Arq Int Otorrinolaringol. 2006;10(3):192-6.
20. Manubens RS. Predisposição individual nas perdas auditivas induzidas pelo ruído ocupacional - PAIRO. Rev CIPA.1995;15:189. 